University of Nebraska - Lincoln

DigitalCommons@University of Nebraska - Lincoln

Robert Streubel Papers

Research Papers in Physics and Astronomy

$9-14-2015$

\title{
Magnetization dynamics of imprinted non-collinear spin textures
}

\author{
Robert Streubel \\ Leibniz-Institut für Festkörper- und Werkstoffforschung Dresden, streubel@unl.edu \\ Peter Fischer \\ Lawrence Berkeley National Laboratory \\ Martin Kopte \\ Leibniz-Institut für Festkörper- und Werkstoffforschung Dresden \\ Oliver G. Schmidt \\ Leibniz-Institut für Festkörper- und Werkstoffforschung Dresden \\ Denys Makarov \\ Leibniz-Institut für Festkörper- und Werkstoffforschung Dresden
}

Follow this and additional works at: https://digitalcommons.unl.edu/physicsstreubel

Part of the Atomic, Molecular and Optical Physics Commons, Condensed Matter Physics Commons, and the Other Physics Commons

Streubel, Robert; Fischer, Peter; Kopte, Martin; Schmidt, Oliver G.; and Makarov, Denys, "Magnetization dynamics of imprinted non-collinear spin textures" (2015). Robert Streubel Papers. 18.

https://digitalcommons.unl.edu/physicsstreubel/18

This Article is brought to you for free and open access by the Research Papers in Physics and Astronomy at DigitalCommons@University of Nebraska - Lincoln. It has been accepted for inclusion in Robert Streubel Papers by an authorized administrator of DigitalCommons@University of Nebraska - Lincoln. 


\section{Magnetization dynamics of imprinted non- collinear spin textures}

Cite as: Appl. Phys. Lett. 107, 112406 (2015); https://doi.org/10.1063/1.4931101

Submitted: 17 June 2015 • Accepted: 04 September 2015 • Published Online: 18 September 2015

Robert Streubel, Peter Fischer, Martin Kopte, et al.

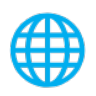

View Online

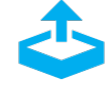

\section{ARTICLES YOU MAY BE INTERESTED IN}

The design and verification of MuMax3

AIP Advances 4, 107133 (2014); https://doi.org/10.1063/1.4899186

Perspective: Magnetic skyrmions-Overview of recent progress in an active research field Journal of Applied Physics 124, 240901 (2018); https://doi.org/10.1063/1.5048972

Generation and stability of structurally imprinted target skyrmions in magnetic multilayers Applied Physics Letters 115, 112404 (2019); https://doi.org/10.1063/1.5099991

\section{Challenge us.}

What are your needs for periodic signal detection?

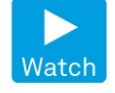

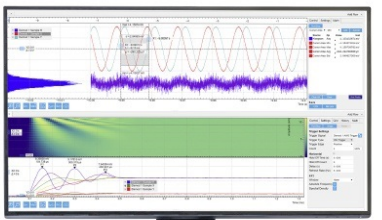

-
Zurich

- Instruments 


\title{
Magnetization dynamics of imprinted non-collinear spin textures
}

\author{
Robert Streubel, ${ }^{1, a)}$ Peter Fischer, ${ }^{2,3}$ Martin Kopte, ${ }^{1}$ Oliver G. Schmidt, ${ }^{1,4}$ \\ and Denys Makarov ${ }^{1, b)}$ \\ ${ }^{1}$ Institute for Integrative Nanosciences, IFW Dresden, 01069 Dresden, Germany \\ ${ }^{2}$ Center for X-Ray Optics, Lawrence Berkeley National Laboratory, Berkeley, California 94720, USA \\ ${ }^{3}$ Physics Department, UC Santa Cruz, Santa Cruz, California 95064, USA \\ ${ }^{4}$ Material Systems for Nanoelectronics, Chemnitz University of Technology, 09107 Chemnitz, Germany
}

(Received 17 June 2015; accepted 4 September 2015; published online 18 September 2015)

\begin{abstract}
We study the magnetization dynamics of non-collinear spin textures realized via imprint of the magnetic vortex state in soft permalloy into magnetically hard out-of-plane magnetized $\mathrm{Co} / \mathrm{Pd}$ nanopatterned heterostructures. Tuning the interlayer exchange coupling between soft- and hardmagnetic subsystems provides means to tailor the magnetic state in the $\mathrm{Co} / \mathrm{Pd}$ stack from being vortex- to donut-like with different core sizes. While the imprinted vortex spin texture leads to the dynamics similar to the one observed for vortices in permalloy disks, the donut-like state causes the appearance of two gyrofrequencies characteristic of the early and later stages of the magnetization dynamics. The dynamics are described using the Thiele equation supported by the full scale micromagnetic simulations by taking into account an enlarged core size of the donut states compared to magnetic vortices. @ 2015 AIP Publishing LLC. [http://dx.doi.org/10.1063/1.4931101]
\end{abstract}

Chiral magnetic spin textures, such as magnetic spin helices and skyrmions, ${ }^{1,2}$ stabilized by Dzyaloshinskii-Moriya interaction $(\mathrm{DMI})^{3,4}$ in single crystals ${ }^{5-8}$ or at interfaces ${ }^{9-11}$ with inversion symmetry breaking are subject to intense fundamental research. The inertia of skyrmion lattices under external perturbations ${ }^{12-14}$ combined with ultra-low spin-current densities needed for coherent displacement make them appealing for application as low-energy spintronic devices. ${ }^{15}$ In general, the spin-orbit interaction governing DMI is weak compared to the Heisenberg exchange and favors chiral spin textures only within a small pocket phase with respect to temperature and magnetic bias field. ${ }^{1,2,5-11}$ Therefore, much attention was devoted to realizing skyrmionic textures in thin film heterostructures providing interfacial $\mathrm{DMI}^{9-11}$ or interlayer exchange interaction. ${ }^{16-19}$ The latter group, referred to as artificial skyrmions ${ }^{17,18}$ due to absent DMI, provides means to stabilize chiral spin textures including skyrmionic core textures at room temperature and remanence. Interestingly, topological charge/ skyrmion number can be switched in a digital manner between 0 and $1,{ }^{18,19}$ paving the way towards spintronics operating on topological properties of magnetic nano-objects. Whereas static properties of such spin textures are well understood, dynamic properties are yet to be investigated. Here, we probe the magnetization dynamics of chiral spin textures formed via imprinting magnetic vortices from Permalloy $\left(\mathrm{Py}, \mathrm{Ni}_{80} \mathrm{Fe}_{20}\right)$ disks into out-of-plane magnetized $\mathrm{Co} / \mathrm{Pd}$ multilayer stacks. ${ }^{19}$

Coplanar waveguides (CPW) consisting of $100 \mathrm{~nm}$-thick and $10 \mu \mathrm{m}$-wide $\mathrm{Cu}$ wires with an impedance $Z \approx 50 \Omega$ were fabricated onto $200 \mathrm{~nm}$-thin $\mathrm{Si}_{3} \mathrm{~N}_{4}$ membranes using optical lithography and sputter deposition. PMMA photoresist is spin-coated on top and patterned via electron beam lithography leaving behind circular holes with diameters of $\varnothing=$ $(650 \pm 20) \mathrm{nm}$ and $\varnothing=(800 \pm 20) \mathrm{nm}$ on the CPW. Afterwards, layer stacks consisting of $\mathrm{Pd}(2) /[\mathrm{Co}(0.4) /$

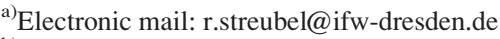

b)Electronic mail: d.makarov@ifw-dresden.de
}

$\operatorname{Pd}(0.7)]_{5} / \mathrm{Pd}(d) / \mathrm{Ni}_{80} \mathrm{Fe}_{20}(40) / \mathrm{Pd}(2)$ with units in $\mathrm{nm}$ and a Pd spacer thickness $d=1 \mathrm{~nm}, d=5 \mathrm{~nm}$ were deposited via $\mathrm{dc}$ magnetron sputtering at room temperature (base pressure: $7 \times 10^{-8} \mathrm{mbar}$; Ar pressure: $10^{-3} \mathrm{mbar}$, rate: $\left.1 \AA / \mathrm{s}\right)$. The magnetic nanostructures [Fig. 1(a)] were obtained after lifting off the PMMA resist. Thicknesses of individual Co and $\mathrm{Pd}$ layers in the $\mathrm{Co} / \mathrm{Pd}$ stack were chosen to induce a large out-of-plane magnetic anisotropy and full remanence. ${ }^{19}$ The Pd spacer thickness separating Permalloy $\left(\mathrm{Py}, \mathrm{Ni}_{80} \mathrm{Fe}_{20}\right)$ and $\mathrm{Co} / \mathrm{Pd}$ stack is adjusted to provide strong $(d=1 \mathrm{~nm})$ and moderate $(d=5 \mathrm{~nm})$ interlayer exchange coupling. We showed in a previous work $^{19}$ that systems with strong

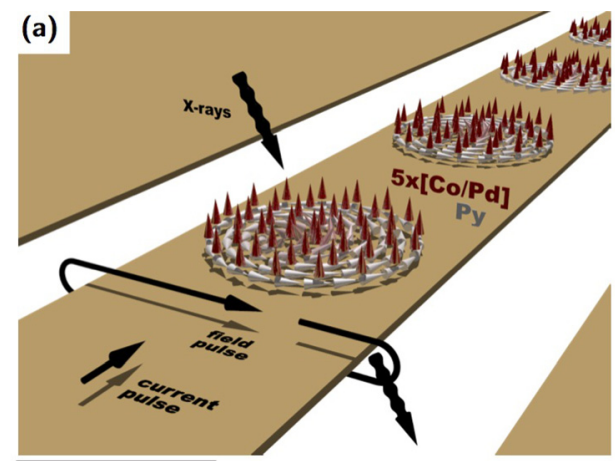

(b) Co/Pd spins
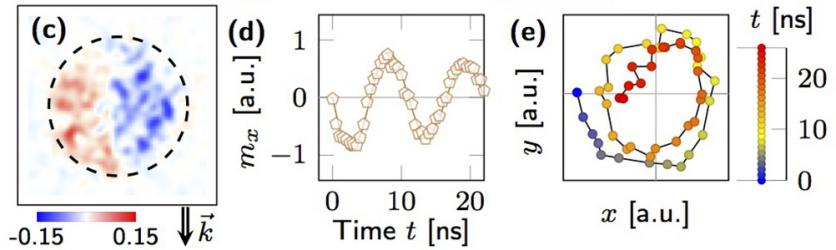

FIG. 1. (a) Vertically stacked soft-magnetic (Py, $\left.\mathrm{Ni}_{80} \mathrm{Fe}_{20}\right)$ and hardmagnetic $(\mathrm{Co} / \mathrm{Pd})$ disks prepared onto a coplanar waveguide are excited with current pulses and stroboscopically recorded in MTXM. (b) Simulated spin configuration in $\mathrm{Co} / \mathrm{Pd}$ multilayer stacks. (c) Visualization of in-plane magnetization component of the Py subsystem utilizing XMCD. (d) Extracted oscillations of the magnetization component that are correlated to the vortex core displacement (e). 
interlayer interaction reveal a magnetic vortex state imprinted into the $\mathrm{Co} / \mathrm{Pd}$ subsystem [Fig. 1(b), top], whereas moderate coupling stabilizes magnetic donut states with either one or two circular domain walls that are referred to as donut state type I [Fig. 1(b), middle] and type II [Fig. 1(b), middle], respectively. $40 \mathrm{~nm}$-thick Py disks with diameters of $\varnothing=(1100 \pm 20) \mathrm{nm}$ and $\varnothing=(1750 \pm 20) \mathrm{nm}$ were prepared for reference.

The magnetization dynamics were studied with magnetic transmission soft X-ray microscopy (MTXM) at the Advanced Light Source (ALS, beamline 6.1.2) in the twobunch mode. The sample is tilted by $30^{\circ}$ with respect to the $\mathrm{X}$-ray beam propagation direction along a rotation axis coinciding with the CPW. Thus, the in-plane magnetization can be accessed utilizing X-ray magnetic circular dichroism (XMCD) as element specific contrast mechanism. We observe the typical dipolar XMCD contrast when imaging Py disks at the $\mathrm{Fe} L_{3}$ absorption edge, which refers to a magnetic vortex state in the reference samples [Fig. 1(c)]. The magnetization dynamics in these patterned nanostructures are stroboscopically recorded synchronizing electric current pulses $(I \approx 100 \mathrm{~mA}, \Delta t=1 \mathrm{~ns})$ from a pulse generator with the electron bunches orbiting in the synchrotron storage ring. The corresponding in-plane magnetic Oersted field with an amplitude of about $8 \mathrm{kA} / \mathrm{m}$ shifts the vortex core by $\approx 30 \%$ of the diameter towards the edge of the disk. The annihilation of the Py vortex is observed at $12 \mathrm{kA} / \mathrm{m}$ when applying static in-plane fields.

For the reference Py sample, the in-plane oscillation of the magnetization component along the direction of the field excitation is shown in Fig. 1(d). This data can be represented as the displacement of the magnetic vortex core assuming a harmonic oscillation with the gyrofrequency $\omega_{0}$ [Fig. 1(e)]. The dynamics of the normalized longitudinal magnetization component are accordingly described by

$$
m_{x}(\varepsilon, t)=\sin \left[2 \pi \omega_{0}(\varepsilon) t\right] \cdot \exp (-\lambda t),
$$

with an aspect ratio $\varepsilon$ defined as the quotient of Py film thickness $h=40 \mathrm{~nm}$ and disk diameter $\varnothing$, and phenomenological damping factor $\lambda=\alpha \gamma / M_{S} \approx \alpha \times 176 \mathrm{GHz}$ with Gilbert damping factor $\alpha$ and gyromagnetic ratio of an isolated electron $\gamma=2 \pi \times 28 \mathrm{GHz} / \mathrm{T}$. Using (1), the magnetization dynamics are fitted with $\omega_{0}(\varepsilon=0.02) \approx 0.09 \mathrm{GHz}$ and $\omega_{0}(\varepsilon=0.04)$ $\approx 0.14 \mathrm{GHz}$ as well as $\alpha \approx 2 \times 10^{-4}$ [Fig. 1(d); only data for $\varepsilon=0.02$ is shown], which is in agreement with previous observations. ${ }^{20,21}$

The total Co thickness of the Co/Pd subsystem of $2 \mathrm{~nm}$ is insufficient to record a detectable signal at the $\mathrm{Co} L_{3}$ edge due to the limited X-ray intensity of the two-bunch mode. In the following, we study the impact of the imprinted magnetic state in the $\mathrm{Co} / \mathrm{Pd}$ subsystem on the magnetization dynamics of the Py spins. All images are recorded at the $\mathrm{Fe} L_{3}$ absorption edge. Figure 2 plots the magnetization oscillations extracted from stroboscopically recorded images [Fig. 2(a)] for disks with different aspect ratios consisting of coupled $[\mathrm{Co} / \mathrm{Pd}] / \mathrm{Pd} / \mathrm{Py}$ stacks with $1 \mathrm{~nm}$ - and $5 \mathrm{~nm}$-thick Pd spacer. The strongly coupled system with $d=1 \mathrm{~nm}$ reveals a harmonic oscillation with one gyrofrequency similar to pure Py [Fig. 2(b)]. Samples in the donut state $(d=5 \mathrm{~nm})$ reveal a
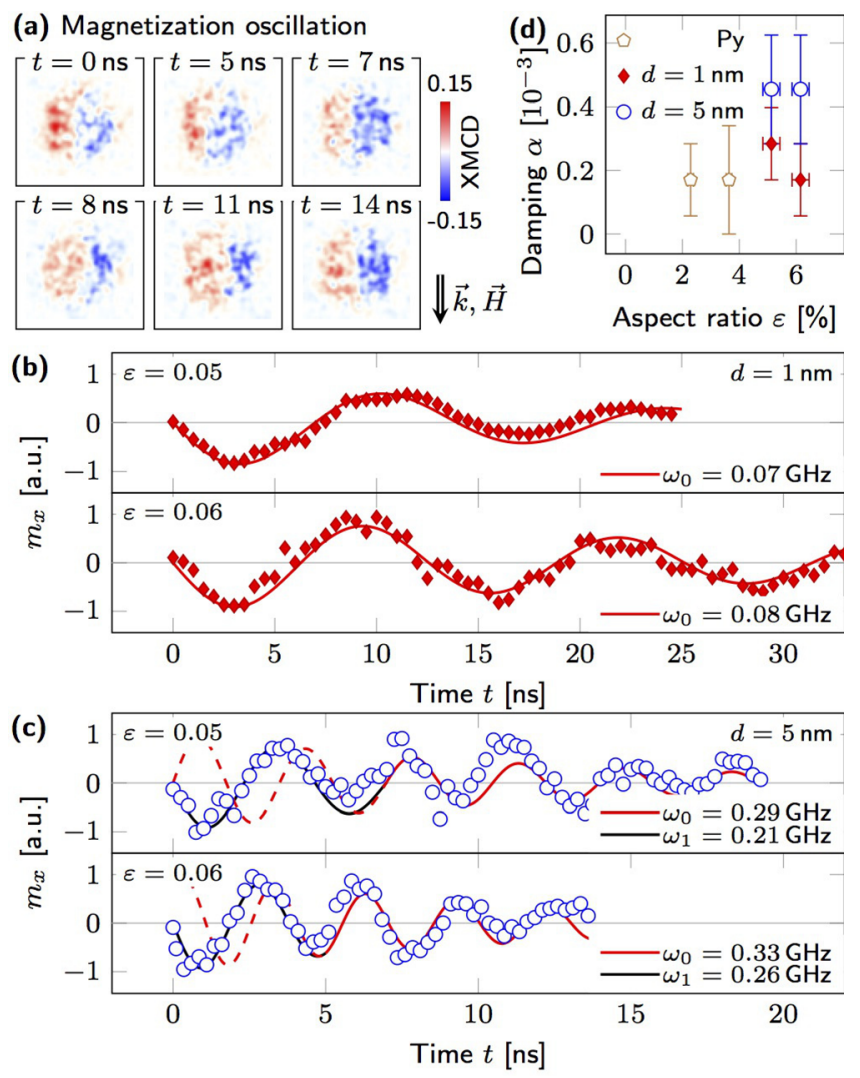

FIG. 2. Time dependence of the in-plane magnetization component of Py along the field direction of the magnetic field pulse measured with MTXM. (a) Stroboscopically recorded XMCD contrast patterns. (b) Strongly coupled systems $(d=1 \mathrm{~nm})$ with two different aspect ratios $\varepsilon$ reveal gyrofrequencies $\omega_{0} \approx 0.08 \mathrm{GHz}$. (c) Moderately coupled systems $(d=5 \mathrm{~nm})$ possess a timedependent gyrofrequency that increases rapidly after about one period. (d) Gilbert damping factors for coupled and reference systems.

three times larger frequency than those of strongly coupled stacks $(d=1 \mathrm{~nm})[$ Fig. 2(c)]. Moreover, the relaxation process cannot be described with one single frequency using (1). For instance, samples with an aspect ratio $\varepsilon=0.05$ reveal in the first $\approx 7 \mathrm{~ns}$ a gyrofrequency $\omega_{1} \approx(0.20 \pm 0.02) \mathrm{GHz}$ and a gyrofrequency $\omega_{0} \approx(0.30 \pm 0.02) \mathrm{GHz}$ afterwards. Furthermore, the damping is lowest for pure Py with inplane magnetization and increases with decreasing interlayer exchange coupling [Fig. 2(d)]. These dynamics are distinct from typical vortex core dynamics in Py disks.

To understand the physical origin, we first perform micromagnetic simulations at $T=0 \mathrm{~K}$ using Nmag v. $0.2,{ }^{22}$ the HLib library ${ }^{23}$ and typical materials parameters for $\mathrm{Co} / \mathrm{Pd}$ ( $h=5 \mathrm{~nm}, M_{S}=500 \mathrm{kA} / \mathrm{m}, A=10^{-11} \mathrm{~J} / \mathrm{m}$, and $K=200 \mathrm{~kJ} /$ $\left.\mathrm{m}^{3}\right)$ and Py $\left(h=40 \mathrm{~nm}, M_{S}=860 \mathrm{kA} / \mathrm{m}\right.$, and $A=1.3 \times 10^{-11}$ $\mathrm{J} / \mathrm{m}$ ). The modeled disks possess a diameter of $400 \mathrm{~nm}$. The size distribution of the tetrahedral mesh resembles a Poisson distribution with peak and mean values around $6 \mathrm{~nm}^{3}$ and $22 \mathrm{~nm}^{3}$, respectively. The standard deviation is about $40 \mathrm{~nm}^{3}$. The interlayer exchange coupling strength is varied in the range $J_{i}=(0.4-1) \mathrm{mJ} / \mathrm{m}^{2}$. Note that the interlayer exchange coupling through the $1 \mathrm{~nm}$-thick and $5 \mathrm{~nm}$-thick Pd spacer is mediated by Ruderman-Kittel-Kasuya-Yoshida (RKKY)-type coupling and by spin diffusion mechanisms involving unfilled $4 d$ and $5 s-p$ Pd bands, ${ }^{24}$ respectively. Landau-LifschitzGilbert damping coefficients of $\alpha_{P y}=0.05$ and $\alpha_{C o}=0.1$ are used for efficient computation. Simulations with damping 
coefficients one order of magnitude smaller than those back up our results revealing immune dynamics with similar gyrofrequencies and trajectories. Line profile of the normal magnetization component and core sizes of donut states imprinted into the $\mathrm{Co} / \mathrm{Pd}$ subsystem are shown in Figs. 3(a) and 3(b), respectively. For comparison, the normal magnetization component of ordinary out-of-plane magnetized bubble domains in plotted as blue curve in Fig. 3(a). In both cases, the circular domain wall is located at $\frac{1}{2 \sqrt{2}} \varnothing$ providing a balanced contribution of negative and positive magnetostatic surface charges. Both core sizes and normal magnetization components can be continuously tuned by adjusting the interlayer coupling through the non-magnetic Pd spacer. Comparing numerical core size with experimentally determined values suggests that a Pd spacer thickness of $d=5 \mathrm{~nm}$ mediates an interlayer exchange coupling of about $0.4 \mathrm{~mJ} / \mathrm{m}^{2} .{ }^{19}$ While the core size of the $\mathrm{Co} / \mathrm{Pd}$ layers can be as large as $100 \mathrm{~nm}$ for weak coupling, the Py vortex core remains unaffected with an almost thickness-independent profile [Fig. 3(c)]. This is because the vortex core dimension is determined by the unmodified exchange, while the core size of the donut state is adjusted according to the dipole interaction and thus the normal magnetization component that is tailored by the interlayer exchange coupling. This peculiarity suggests the key role of the $\mathrm{Co} / \mathrm{Pd}$ core on the magnetization dynamics of the entire system.

In order to obtain further insight, the relaxation process is analytically described using the Thiele equation for magnetic domain motion ${ }^{25}$

$$
\mathcal{G} e_{z} \times \dot{\boldsymbol{r}}-\mathcal{K} \boldsymbol{r}=\mathcal{M} \ddot{\boldsymbol{r}}
$$

with $\boldsymbol{r}=(x, y)$. Approximating the donut state as an out-ofplane magnetized bubble domain, the coefficients represent
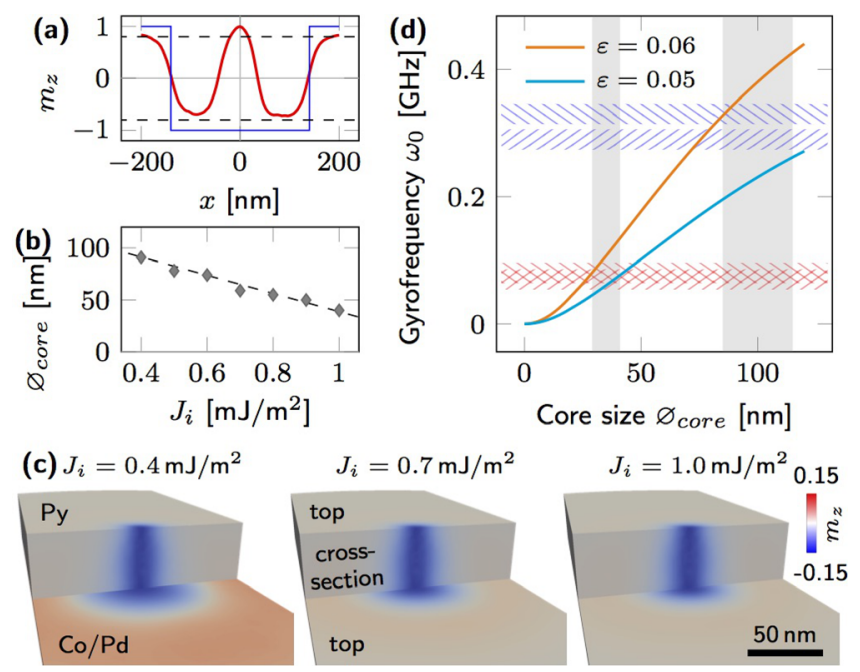

FIG. 3. (a) Profiles of the normal magnetization component across the $\mathrm{Co} / \mathrm{Pd}$ layer obtained from micromagnetic simulations using $J_{i}=0.4 \mathrm{~mJ} / \mathrm{m}^{2}$ (red curve) and $J_{i}=0 \mathrm{~mJ} / \mathrm{m}^{2}$ (blue curve). (b) Numerically calculated core size as a function of interlayer exchange coupling. (c) Cross-section of the normal magnetization component through Py vortex core and top view of $\mathrm{Co} / \mathrm{Pd}$ magnetization for various interlayer coupling strengths. (d) Analytical calculation of the relation between gyrofrequency and core size of the $\mathrm{Co} / \mathrm{Pd}$ stack. Experimentally determined gyrofrequencies are indicated by horizontally shaded areas (red $(\mathrm{d}=1 \mathrm{~nm}): \omega_{0}=(0.07 \pm 0.2) \mathrm{GHz}$ and $(0.08 \pm 0.2) \mathrm{GHz}$; blue $(\mathrm{d}=5 \mathrm{~nm}): \omega_{0}=(0.29 \pm .02) \mathrm{GHz}$ and $\left.(0.33 \pm 0.2) \mathrm{GHz}\right)$. Core sizes for strong $[(35 \pm 6) \mathrm{nm}]$ and moderate $[(100 \pm 12) \mathrm{nm}]$ coupling obtained by micromagnetic simulations are indicated by vertical gray shaded areas. the gyrotropic coefficient $\mathcal{G} \propto S h$, the restoring force $\mathcal{K} \propto M_{z}^{2} \frac{h^{2}}{\varnothing} \exp \left(\varnothing_{\text {core }}\right)$, and the core mass $\mathcal{M} \propto \mathcal{G}^{2}$ $\varnothing_{\text {core }}{ }^{26,27} S$ is the skyrmion number $S=\frac{1}{4 \pi} \int \boldsymbol{m}\left(\partial_{x} \boldsymbol{m}\right.$ $\left.\times \partial_{y} \boldsymbol{m}\right) \mathrm{d} x \mathrm{~d} y$ with the normalized magnetization $\boldsymbol{m}$, which becomes $S= \pm \frac{1}{2}$ and $S= \pm 1$ for vortex states and bubble domains, respectively. Donut states with an interlayer exchange coupling of about $0.4 \mathrm{~mJ} / \mathrm{m}^{2}$ possess $S \approx \pm 0.9$ (type I) and $S \approx \pm 0.1$ (type II). ${ }^{19}$ The size of vortex cores in Py of about $6 \mathrm{~nm}$ lead to particle-like dynamics that can be described without the mass term $(\mathcal{M}=0)$ resulting in the well-known relationship between aspect ratio and gyrofrequency $\omega_{0}=\mathcal{K} / \mathcal{G} \propto \frac{h}{\varnothing S}{ }^{20,21}$ This simplification is no longer valid for donut states with extended cores of $\varnothing_{\text {core }} \approx$ $100 \mathrm{~nm}$ [Fig. 3(b)]. The major contributions of restoring force $\mathcal{K}$ and core mass $\mathcal{M}$ cause an increase of the gyrofrequency with increasing core size $\varnothing_{\text {core }}$ or disk diameter $\varnothing$. This trend is quantitatively confirmed [Fig. 3(d)], using the exact expressions for $\mathcal{K}, \mathcal{M}$, and $\mathcal{G}$ given in Refs. 26 and 27 with properly modified saturation magnetization and restoring force. The saturation magnetization of the coupled systems is approximated as the mean value of both subsystems weighted by their thicknesses $M=\left(\frac{8}{9} \cdot 860+\frac{1}{9} \cdot 500\right) \mathrm{kA} / \mathrm{m}$ $\left(M_{\mathrm{Py}}=860 \mathrm{kA} / \mathrm{m} ; h_{\mathrm{Py}}=40 \mathrm{~nm} ; M_{\mathrm{Co} / \mathrm{Pd}}=500 \mathrm{kA} / \mathrm{m} ; h_{\mathrm{Co} / \mathrm{Pd}}\right.$ $=5 \mathrm{~nm}$ ). The restoring force $\mathcal{K}$ originally calculated for outof-plane magnetized bubble domains is rescaled $\mathcal{K} \rightarrow 0.64 \mathcal{K}$ taking into account reduced magnetostatic surface charges due to oblique magnetization vectors with normal magnetization components of about $0.8 M_{S}$ in the $\mathrm{Co} / \mathrm{Pd}$ subsystem with $d=5 \mathrm{~nm}\left(J_{i}=0.4 \mathrm{~mJ} / \mathrm{m}^{2}\right)$ [Fig. 3(a), red curve].

The localization of the out-of-plane magnetization components in vortex cores with sizes of about $6 \mathrm{~nm}$ implies a solitary motion that can be described by one gyrofrequency [Fig. 1(d)]. Similarly, this approximation holds for strongly coupled systems with core sizes $\varnothing_{\text {core }}(d=1 \mathrm{~nm}) \approx 35 \mathrm{~nm}$ [Fig. 2(b)]. In contrast, donut states with extended cores $\varnothing_{\text {core }}(d=$ $5 \mathrm{~nm}) \approx 100 \mathrm{~nm}$ reveal two distinct gyrofrequencies, which
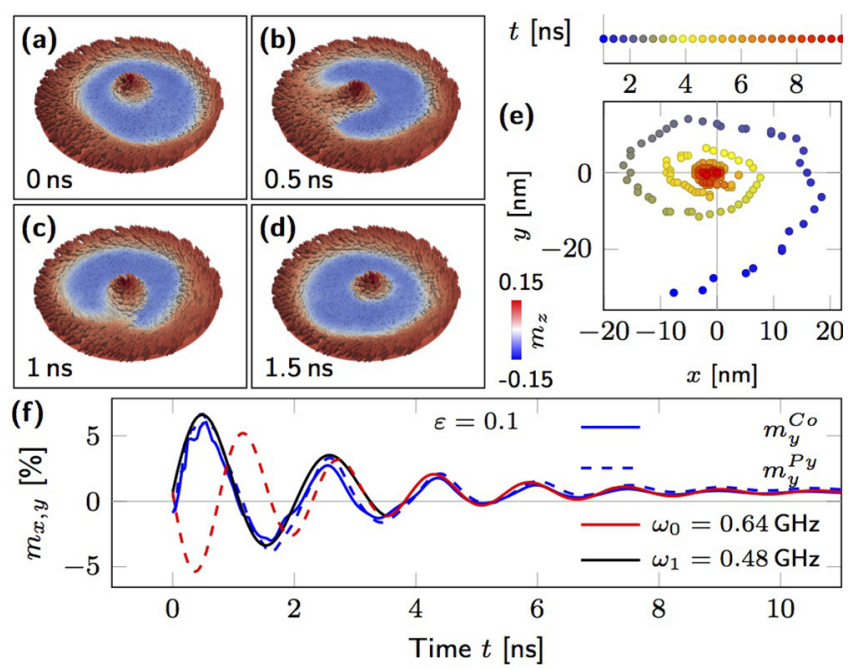

FIG. 4. Simulated relaxation of Co/Pd multilayers coupled to a Py vortex state via an interlayer exchange strength of $J_{i}=0.4 \mathrm{~mJ} / \mathrm{m}^{2}$ after applying an in-plane magnetic field of $8 \mathrm{kA} / \mathrm{m}$. (a)-(d) Snapshots. Red and blue contrasts refer to normal magnetization components. The donut state temporarily transforms into a " $\mathrm{C}$ " state (b)-(d) causing a domain wall vibration and (e) kinks in the trajectory of the vortex core as well as (f) a time-dependent gyrofrequency similar to those observed in experiment. 
originate from a temporary deformation of the core in the first couple of nanoseconds [Fig. 4]. This instability may be caused by an interaction with the edge of the disk or by a dynamically excited vibration of the domain wall. The accordingly affected core dynamics reveal a trajectory with kinks [Fig. 4(e)] similar to those reported for bubble domains, ${ }^{27}$ which justifies the use of analytical expressions derived for bubble domains with dynamic mass term to treat the motion of donut states.

In conclusion, we have presented the magnetization dynamics of imprinted non-collinear chiral spin textures recorded with transmission soft X-ray microscopy. A clear dependence of the gyrofrequency on the interlayer exchange coupling strength was observed that offers an indirect access to the core diameter via its dynamics. Moreover, understanding magnetization dynamics of skyrmionic core textures in view of field- and current-driven displacements is an essential ingredient to realize memory and logic devices.

We thank I. Fiering and I. Mönch (both IFW Dresden) for metal deposition and assistance with electron beam lithography, respectively. V. P. Kravchuk (BITP Kiev) is acknowledged for fruitful discussions on the analytical treatment. This work was financed via the ERC under EU's 7th FP (FP7/2007-2013)/ERC Grant Agreement No. 306277. P.F. acknowledges support from DOE BES MSED (DEAC02-05-CH11231) and by Leading Foreign Research Institute Recruitment Program (2012K1A4A3053565) through the NRF of Korea funded by MEST.

${ }^{1}$ A. N. Bogdanov and D. A. Yablonskii, Sov. Phys. JETP 68, 101 (1989), available at http://www.jetp.ac.ru/cgi-bin/e/index/e/68/1/p101?a=list.

${ }^{2}$ U. K. Roessler, A. N. Bogdanov, and C. Pfleiderer, Nature (London) 442, 797 (2006).

${ }^{3}$ I. E. Dzyaloshinskii, Sov. Phys. JETP 5, 1259 (1957).

${ }^{4}$ T. Moriya, Phys. Rev. 120, 91 (1960).
${ }^{5}$ X. Z. Yu, Y. Onose, N. Kanazawa, J. H. Park, J. H. Han, Y. Matsui, N. Nagaosa, and Y. Tokura, Nature (London) 465, 901 (2010).

${ }^{6}$ N. Kanazawa, Y. Onose, T. Arima, D. Okuyama, K. Ohoyama, S. Wakimoto, K. Kakurai, S. Ishiwata, and Y. Tokura, Phys. Rev. Lett. 106, 156603 (2011).

${ }^{7}$ S. X. Huang and C. L. Chien, Phys. Rev. Lett. 108, 267201 (2012).

${ }^{8}$ S. Seki, X. Z. Yu, S. Ishiwata, and Y. Tokura, Science 336, 198 (2012).

${ }^{9}$ A. N. Bogdanov and U. K. Rößler, Phys. Rev. Lett. 87, 037203 (2001).

${ }^{10}$ S. Heinze, K. von Bergmann, M. Menzel, J. Brede, A. Kubetzka, R. Wiesendanger, G. Bihlmayer, and S. Blugel, Nat. Phys. 7, 713 (2011).

${ }^{11}$ N. Romming, C. Hanneken, M. Menzel, J. E. Bickel, B. Wolter, K. von Bergmann, A. Kubetzka, and R. Wiesendanger, Science 341, 636 (2013).

${ }^{12}$ F. Jonietz, S. Mühlbauer, C. Pfleiderer, A. Neubauer, W. Münzer, A. Bauer, T. Adams, R. Georgii, P. Böni, R. A. Duine, K. Everschor, M. Garst, and A. Rosch, Science 330, 1648 (2010).

${ }^{13}$ X. Yu, N. Kanazawa, W. Zhang, T. Nagai, T. Hara, K. Kimoto, Y. Matsui, Y. Onose, and Y. Tokura, Nat. Commun. 3, 988 (2012).

${ }^{14}$ M. Mochizuki, X. Z. Yu, S. Seki, N. Kanazawa, W. Koshibae, J. Zang, M. Mostovoy, Y. Tokura, and N. Nagaosa, Nat. Mater. 13, 241 (2014).

${ }^{15}$ A. Fert, V. Cros, and J. Sampaio, Nat. Nano 8, 152 (2013).

${ }^{16}$ Y. Y. Dai, H. Wang, P. Tao, T. Yang, W. J. Ren, and Z. D. Zhang, Phys. Rev. B 88, 054403 (2013).

${ }^{17}$ L. Sun, R. X. Cao, B. F. Miao, Z. Feng, B. You, D. Wu, W. Zhang, A. Hu, and H. F. Ding, Phys. Rev. Lett. 110, 167201 (2013).

${ }^{18}$ J. Li, A. Tan, K. Moon, A. Doran, M. Marcus, A. Young, E. Arenholz, S. Ma, R. Yang, C. Hwang, and Z. Qiu, Nat. Commun. 5, 4704 (2014).

${ }^{19}$ R. Streubel, L. Han, M.-Y. Im, F. Kronast, U. K. Rößler, F. Radu, R. Abrudan, G. Lin, O. G. Schmidt, P. Fischer, and D. Makarov, Sci. Rep. 5, 8787 (2015).

${ }^{20}$ K. Y. Guslienko, X. F. Han, D. J. Keavney, R. Divan, and S. D. Bader, Phys. Rev. Lett. 96, 067205 (2006).

${ }^{21}$ Y. Gaididei, V. P. Kravchuk, and D. D. Sheka, Int. J. Quantum Chem. 110, 83 (2010).

${ }^{22}$ T. Fischbacher, F. Matteo, G. Bordignon, and H. Fangohr, IEEE Trans. Magn. 43, 2896 (2007).

${ }^{23} \mathrm{~W}$. Hackbusch, Hierarchische Matrizen: Algorithmen und Analysis (Springer, 2009).

${ }^{24}$ Z. Celinski, B. Heinrich, J. F. Cochran, W. B. Muir, A. S. Arrott, and J. Kirschner, Phys. Rev. Lett. 65, 1156 (1990).

${ }^{25}$ A. A. Thiele, Phys. Rev. Lett. 30, 230 (1973).

${ }^{26}$ I. Makhfudz, B. Krüger, and O. Tchernyshyov, Phys. Rev. Lett. 109, 217201 (2012).

${ }^{27}$ K.-W. Moon, B. S. Chun, W. Kim, Z. Q. Qiu, and C. Hwang, Phys. Rev. B 89, 064413 (2014). 